\title{
Why videofluoroscopy?
}

\section{A R Lloyd-Jones MComm Path \\ Department of Speech Therapy and Audiology Pretoria Academic Hospital}

Swallowing may be defined as a highly complex and co-ordinated sequence of neuromuscular events designed to transport food and liquids from the oral cavity, through the pharynx and oesophagus, to the stomach. During this process, the respiratory function ceases briefly to allow the digestive function to take place and resumes once the swallowing sequence has been completed. ${ }^{1}$ When this complex sequence of events becomes disordered and the transportation of material is no longer efficient or effective, then dysphagia is present.

Dysphagia may manifest in varying degrees, from mild symptoms such as the sensation of food sticking in the throat, to severe dysphagia where the oral intake of food or liquids is impossible. Dysphagia can also occur at any stage of life, from the premature infant who cannot suck and swallow, to the geriatric patient, and can have a profound effect on the quality of life experienced. Symptoms such as coughing and choking, drooling, nasal regurgitation and oesophageal reflux may cause the sufferer to withdraw from society and experience feelings of isolation and depression. ${ }^{2}$ In addition, dysphagia carries the sequelae of malnutrition, dehydration and aspiration if left undiagnosed and untreated. ${ }^{3}$

In view of the serious mortality and morbidity associated with dysphagia it is important to differentiate between the symptoms of dysphagia and the causes of dysphagia, as the causative factor may be an underlying disease or disorder that may be debilitating or life threatening. ${ }^{4}$ This process of differentiation involves a comprehensive evaluation of the patient and the expertise of a number of medical and allied medical disciplines in order to provide the detailed information that is crucial to diagnosis and management of this condition. ${ }^{5}$ The speech pathologist and the radiologist are essential members of this multidisciplinary dysphagia team.

\section{Evaluation of dysphagia}

As with any patient the evaluation commences with a case history followed by a clinical evaluation but 'the literature clearly indicates that clinical, bedside evaluation is an inadequate and poor predictor of pharyngeal dysphagia.6 Consequently other more objective means of evaluating swallowing, and especially the pharyngeal phase of swallowing, have been sought. As the ability to visualise the phases of swallowing aids in evaluation, radiographic techniques have been used since the early 1900s. The development of fluorography during the 1930s allowed the examination of the movement patterns of the oral cavity, pharynx and oesophagus. With the development of technology the fluorographic technique has been refined and now fluorographic studies are recorded on videotape, reducing the exposure to radiation by making the examination time shorter, and by offering the opportunity for immediate review of the examination.

Another imaging technique that can be used is ultrasonography. Ultrasound clearly visualises muscles, and depicts the surfaces between muscles, organs and blood vessels, and a variety of other tissues as well as the motion of these structures. However, the ultrasound does not visualise bone. ${ }^{8}$ There is a further limitation to ultrasound as not all the phases of swallowing can be visualised, the ultrasound cannot demarcate the bolus after it has passed into the hypopharynx and cannot track the bolus during the oesophageal phase. ${ }^{8}$ Ultrasound does not hold any danger from radiation and can be used over and over again with impunity.

Many other methods of objective evaluation of the swallowing mechanism exist. Of these methods fibreoptic endoscopy coupled with videorecording facilities appear to be the most popular. Manometry, scintigraphy, electromyography and cervical auscultation are also utilised but no other method matches the advantages of videofluoroscopy. Video-fluoroscopy has become known as the gold standard for swallowing evaluation.

\section{Rationale for this choice}

In order to choose the most economical and efficacious method of evaluating swallowing some important factors must be taken into con- 
sideration. The examination should offer the means to track the bolus as well as the motions of the swallowing mechanism, while allowing the ability to observe if any bolus enters the airway and at what time during the swallowing event the aspiration takes place. Another factor to be considered is whether the examination allows for the use of real food bolusses that correspond to a natural diet which would facilitate the management of the dysphagia. If a procedure can be utilised in treatment and follow-up of the dysphagic patient it is an added benefit. ${ }^{8}$

With videofluoroscopy, the procedure produces a clear pictorial image of all the phases of swallowing, namely oral preparatory, oral prepulsive, pharyngeal as well as oesophageal. This procedure provides a complete image of the bony structures of the oropharynx, airway and digestive tract, while the use of high-density barium contrast material outlines the surface and the soft tissue of the tongue, velum, pharynx, larynx and the oesophagus. This means that videofluoroscopy is easy to interpret as it gives a clear likeness of the anatomical structures in their actual form and proportion. In addition, the procedure is dynamic and allows a clear image of the bolus flow through the aerodigestive tract while providing valuable information regarding the patterns of movement as well as the timing of events during swallowing. This makes diagnosing the physiologic and temporospatial components of dysphagia much more accurate and efficient. $^{8}$

With regard to the other factors such as the bolus type and management of dysphagia, videofluoroscopy also offers the most information. The protocol used during videofluoroscopy can utilise food from the normal diet in different textures and consistency in order to examine the effects of the various bolus characteristics on the mechanism of swallowing. Information gained from using different bolus textures will clearly indicate if, for example, a patient aspirates on thin fluids or on a solid particulate bolus. This will aid in managing the dysphagia as the diet of the patient may be manipulated to provide all foods in a texture and consistency compatible with aspiration-free oral feeding. ${ }^{9}$

Further management of swallowing disorders is facilitated by using videofluoroscopy as airway protection manoeuvres and various postural techniques can be used during the examination and the efficacy of the strategy evaluated immediately. The videorecording can be used later as an instructional aid for the patient demonstrating that the techniques work in providing him or her with a safe oral swallow.?

In summary, "Videofluoroscopy is not designed to determine whether someone aspirates, but rather to understand why they aspirate. Further, the study is designed to define optimal eating strategies to enable the patient to continue at least partial intake."

\section{Who to refer}

Any patient who complains of a swallowing disorder should at the very least be referred to a speech therapist who can utilise screening instruments to identify the extent of the problem. This will avoid doing a videofluoroscopy on patients who have mild oral phase dysphagia. However, 'if the main complaint is indicative of respiratory difficulty or suggests the likelihood of aspiration or laryngeal penetration, videofluoroscopy is indicated.

As a result of the varied aetiology of dysphagia which encompasses neurogenic causes, as well as neoplastic and structural causes, the symptoms presented by the patient also indicate whether a videofluoroscopic examination should be performed. A patient who complains of a gradual onset, difficulty in swallowing pills and fluids as well as an increase in eating time probably has a neurogenic disorder as the underlying cause and the complaint should be investigated using videofluoroscopy. A patient who complains of a more sudden onset, painful swallowing, food sticking in the throat and weight loss may have a tumour or a mass and should undergo a videofluoroscopy. ${ }^{8}$

It is important to remember that videofluoroscopy is not an exclusive procedure but that the result obtained from videofluoroscopy will indicate the selection of further tests and procedures to confirm or dispute the findings. It appears to be a common misperception that the videofluoroscopy means that no other tests need to be performed. However, should a tumour or mass be identified further examinations such as a CT scan or MRI will be needed to investigate further. If an oesophageal component is identified during the videofluoroscopy then clear-tests such as manometry, ph monitoring, Ba swallow, etc. are the next investigations required. The videofluoroscopy can aid in diagnosing the swallowing disorder but also saves time and money by indicating which other procedures will be necessary. 


\section{Who should do the videofluo- roscopy?}

As the purpose of a videofluoroscopy is to determine the deficits in the swallowing mechanism that produce the symptoms as well as the therapeutic strategies necessary to compensate for the deficits, it is logical that both a radiologist and a speech therapist should be involved in this procedure.

The radiologist contributes expertise from many fields such as neuroradiology as well the knowledge to identify structural abnormalities. The radiologist is able to help tie together the information from various studies in order to arrive at a diagnosis. Furthermore, the radiologist is responsible for the technical aspects of the procedure such as exposure and the identification and reduction of image artifacts ensuring the quality of the imaging. ${ }^{7.8}$

The speech therapist has specialist knowledge of the movement patterns of the oral structures, pharynx and larynx during deglution and complements the radiologist's knowledge of structural deficits. The speech therapist also has knowledge of the effects of bolus characteristics on swallowing as well as the various postural changes and airway protection manoeuvres. Therefore, she can determine which techniques would facilitate safe oral feeding. In this way the combination of the skills of both these professionals leads to effective and efficient decision-making regarding diagnosis and management of the patient's problem. ${ }^{7}$

In conclusion, videofluoroscopy is a short, relatively cheap, easy-to-interpret test that provides comprehensive information regarding all the phases of swallowing, the deficits and structural defects of the swallowing mechanism and lends itself to the evaluation of the therapeutic techniques employed to overcome the swallowing problem. This procedure involves the combination of the radiologist's skills and the speech therapist's skills to diagnose and manage dysphagia successfully. For in the words of Virginia
Woolf: 'One cannot think well, love well, sleep well, if one has not dined well'.

\section{References}

1. Bradley PJ. Evaluation of the normal swallowing mechanism. CME Bulletin Otorhinolaryngology, Head and Neck Surgery 1998; 2 (1): 4-8.

2. Mathog RH, Fleming SM. A clinical approach to dysphagia. Am J Otolaryngol 1992; 13: 133138.

3. Veldee MS, Peth LD. Can protein-calorie malnutrition cause dysphagia. Dysphagia 1992; 7: 86-101.

4. Fleming SM. Treatment of mechanical swallowing disorders. In: Groher ME, ed. Dysphagia Diagnosis and Management. 2nd ed. Boston: Butterworth-Heineman, 1992: 237-253.

5. Miller RM. Clinical examination for dysphagia. In: Groher ME, ed. Dysphagia. Diagnosis and Management. 2nd ed. Boston: ButterworthHeineman, 1992: 143-163.

6. Leder SB, Sasaki CT, Burrel MI. Fibreoptic endoscopic evaluation of dysphagia to identify silent aspiration. Dysphagia 1988; 13: 19-21.

7. Logeman JA. Evaluation and Treatment of Swallowing Disorders. 2nd ed. Austin, Texas: Pro-Ed, 1998.

8. Sonies BC. Dysphagia: A model for differential diagnosis for adults and children. In: Cherney LR, ed. Clinical Management of Dysphagia in Adults and Children. 2nd ed. Chicago, Illinois: Aspen Publications, 1994.

9. Ekberg O. Evaluation of swallowing. In: Groher ME, ed. Dysphagia Diagnosis and Management. 2nd ed. Boston: Butterworth-Heineman, 1994: 163-196. 